Hydroécol. Appl. (2001) Tome 13 Vol. 2, pp.131-147

\title{
La restauration des poissons migrateurs du bassin de la Loire : l'aménagement des seuils de centrales électriques
}

\author{
The restoration of migratory fish populations \\ on the Loire river: Fish passage facilities \\ for the nuclear power weirs
}

\author{
J-P. Bouchard
}

EDF R\&D, Laboratoire National Hydraulique Environnement, 6, Quai Watier - F 78400 Chatou. Tel : 0130877872 ; fax : 0130878086 jean-pierre.bouchard@edf.fr

Résumé. - Pour assurer l'alimentation en eau de centrales nucléaires implantées en bordure de la Loire, des seuils ont été édifiés. L'abaissement progressif du lit en a fait des obstacles de plus en plus marqués à la migration. L'importance de l'obstacle peut être appréciée par sa hauteur de chute et aussi par l'analyse comparée des vitesses d'écoulement sur le seuil et de la capacité de nage des poissons. La recherche de solutions au problème du franchissement a d'abord reposé sur l'édification de grandes passes à bassins en béton. Les solutions récentes de passes en enrochements cherchent maintenant à utiliser un débit plus important pour favoriser l'attractivité de l'ouvrage et à ménager une grande diversité de conditions d'écoulement pour permettre le passage d'espèces ayant des exigences très différentes.

Mots-clés. - Poissons migrateurs, saumons, aloses, passe à poissons, Loire.

Summary. - A series of 3 weirs has been built on the Loire river to supply nuclear power plants with cooling water. Due to a general trend of bed erosion, these barrages have became an increasing obstacle to fish migration. The importance of the obstacle can be analysed as a function of the fall and of the flow velocity on the weir, to be compared to the swimming ability of fish. Large pools fish passes with vertical slots were firstly built to allow fish migration. More recently a new kind of facility has been proposed, it consists in a series of rockfill pools. In this kind of fish pass, a larger discharge can be used, and the heterogeneity of velocity and water depth enhance passage possibilities for various species.

Key word. - Migratory fish, salmon, shad, fish pass, Loire. 


\section{INTRODUCTION}

L'implantation de centrales en bord d'un fleuve comme la Loire, connu pour la sévérité de ses étiages et l'instabilité de son lit, imposait la construction de seuils pour assurer l'alimentation en eau de refroidissement des installations. Un seuil fut donc construit en aval des centrales de Saint-Laurent des Eaux, Dampierre et Belleville. Dans le cas de Chinon, le fleuve n'est pas coupé par un barrage complet, mais un seuil ferme en étiage un des bras de la Loire pour concentrer l'écoulement dans le chenal de la centrale.

Au total, on trouve donc en Loire moyenne, trois seuils de centrales qui se succèdent sur un bief d'environ $110 \mathrm{~km}$.

Le précédent article de $P$. Steinbach (2002) a décrit le contexte régional des populations de poissons migrateurs et les différents obstacles à leur maintien ou à leur développement. Les seuils de centrales, situés en Loire moyenne, contrôlent l'accès aux zones de reproduction du Massif Central, à l'amont de la confluence avec le Cher. La grande distance de- puis l'estuaire à ces frayères nécessite une remontée rapide de la partie aval du cours d'eau et de ses obstacles pour que le potentiel reproductif de ces zones puisse totalement s'exprimer.

Ces raisons ont justifié l'attention portée aux problèmes du franchissement des seuils de centrales et les aménagements effectués ces dernières années.

\section{NATURE ET IMPORTANCE DES OBSTACLES}

\subsection{Hauteur de chute}

La hauteur de chute est le paramètre le plus simple permettant de caractériser l'obstacle. Les valeurs des chutes sur les trois sites sont rassemblées dans le tableau I ci-dessous.

Quelques remarques sur ces données :

Tout d'abord, l'importance de l'obstacle a augmenté avec le temps. C'est particulièrement net à SaintLaurent où le seuil a été conçu initia-

Tableau I - Caractéristiques des seuils implantés sur la Loire.

Table I - Weirs geometry on the river Loire.

\begin{tabular}{|l|c|c|c|}
\hline & St Laurent & Dampierre & Belleville \\
\hline Année d'édification & 1967 & 1976 & 1979 \\
\hline Chute initiale & $0:$ au niveau des fonds & $<0,8 \mathrm{~m}$ & $<1 \mathrm{~m}$ \\
\hline Chute maximale actuelle (étiage) & $1,85 \mathrm{~m}$ & $1,25 \mathrm{~m}$ & $1,35 \mathrm{~m}$ \\
\hline Chute au débit moyen $\left(350 \mathrm{~m}^{3} / \mathrm{s}\right)$ & $1,55 \mathrm{~m}$ & $1,0 \mathrm{~m}$ & $0,90 \mathrm{~m}$ \\
\hline Chute au débit $600 \mathrm{~m}^{3} / \mathrm{s}$ & $1,30 \mathrm{~m}$ & $0,55 \mathrm{~m}$ & $0,60 \mathrm{~m}$ \\
\hline
\end{tabular}




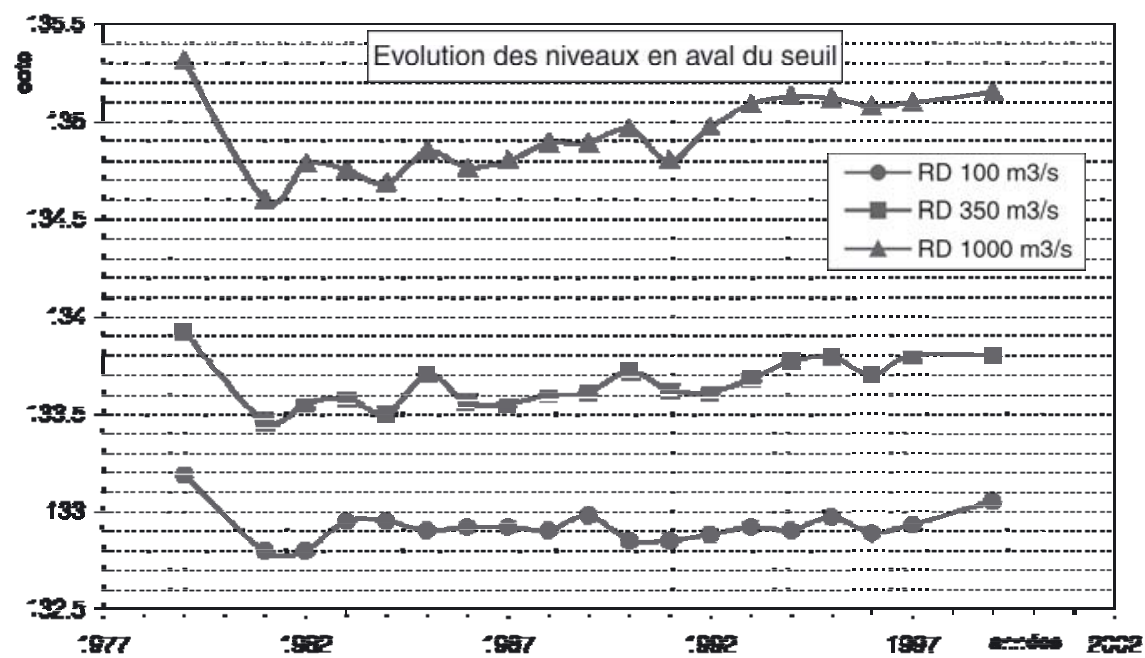

Fig. 1. - Evolution du niveau à l'aval du seuil de Belleville pour trois débits depuis la construction de la centrale.

Fig. 1. - Water table downstream Belleville weir at 3 discharges. Evolution over time since the power plant implementation.

lement à la cote des fonds, puis s'est trouvé dégagé du fait de l'abaissement généralisé du lit du fleuve, selon le même phénomène qui a fait émerger les radiers de pont de la couche d'alluvions. Ce phénomène n'est cependant pas irréversible, ainsi à Belleville la tendance à la remontée du niveau aval du seuil est prouvée : la Figure 1 montre l'évolution au cours du temps des niveaux d'eau à l'aval du seuil. Après un abaissement sensible à la construction de la centrale (1979-1981), on note une remontée régulière particulièrement aux débits forts et moyens.

Le second point est la diminution de la chute avec le débit. Ce mécanisme est plus ou moins marqué seIon les sites, mais il est visible sur chaque seuil et permet l'effacement de l'obstacle pour les plus hauts débits.

\subsection{Nature de l'écoulement sur le seuil}

La chute n'est pas le seul élément qui détermine l'importance de l'obstacle à la migration. La nature de l'écoulement, sur et à l'aval du seuil, participe à rendre la chute plus ou moins franchissable.

A mesure que le niveau aval remonte avec le débit, l'écoulement passe d'un écoulement plongeant très émulsionné, à un écoulement horizontal suivi d'un ressaut plus ou moins marqué. Les photos 1 illustrent bien ces deux types d'écoulements. 

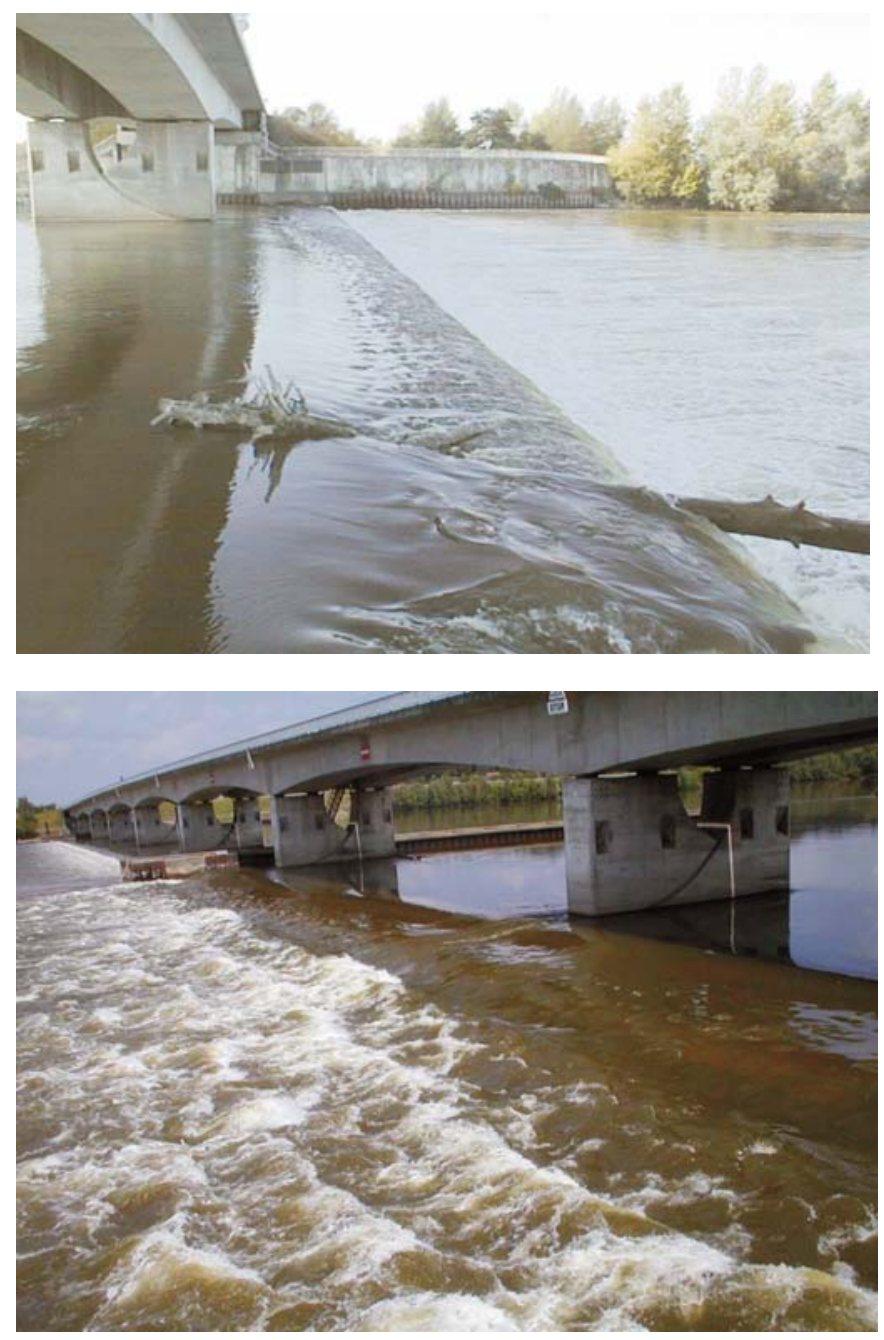

Photos 1 - Ecoulement sur le seuil de Belleville: Débit $125 \mathrm{~m}^{3} / \mathrm{s}$, écoulement plongeant (à gauche) ; Débit $500 \mathrm{~m}^{3} / \mathrm{s}$, écoulement horizontal et ressaut ondulé (à droite).

Photos 1 - Flow conditions over Belleville weir : Left : Flowrate $125 \mathrm{~m}^{3} / \mathrm{s}$, plunging flow. Right : flowrate $500 \mathrm{~m}^{3} / \mathrm{s}$, horizontal flow. with hydraulic jump.

Les écoulements plongeants sont franchissables, lorsque la chute n'est pas trop importante, par les espèces pouvant sauter dans la chute (notamment les salmonidés). Ils forment, par contre, un obstacle pour celles qui doivent franchir la chute en nageant dans la lame d'eau comme les alo- ses ; l'émulsion d'air et d'eau à l'aval de la chute constitue également un facteur de répulsion.

La transition entre écoulement plongeant et écoulement horizontal est donc souvent une condition (non suffisante) pour le passage naturel de 
l'obstacle. Dans le cas du seuil de Belleville cette transition se situe vers $400 \mathrm{~m}^{3} / \mathrm{s}$.

\subsection{Vitesses d'écoulement sur le seuil}

Pour approcher de façon plus quantifiée l'importance de l'obstacle, il faut comparer les capacités de nage des espèces aux conditions hydrauliques générées par le seuil. Cette analyse a été faite pour le seuil de Belleville, où l'on a estimé les vitesses d'écoulement et le tirant d'eau à l'aide d'un calcul simplifié négligeant les pertes de charge par frottement.

Le seuil de Belleville, comme tous les autres seuils de centrales sur la Loire, se présente en coupe selon deux profils différents : un profil tra-

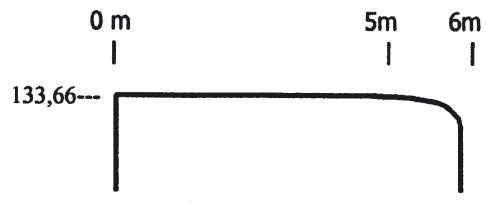

Coupe du seuil coté RG (profil rectangulaire) Left bank weir cross section pézoïdal et un profil rectangulaire plus bas. L'analyse des conditions hydrauliques distingue ces deux parties (Tableau II et Tableau III).

Sur le seuil trapézoïdal (RD), le franchissement est pénalisé par les faibles tirant d'eau et les vitesses très élevées au pied du plan incliné ; sur la partie rectangulaire $(R G)$, les vitesses sont moindres, mais la distance à parcourir est importante (entre 4 et $8 \mathrm{~m}$ selon le point de passage) car le seuil est large.

D'une façon générale, les seuils de centrales sont des seuils épais, ce qui induit un comportement hydraulique particulier : Iorsque le débit en Loire augmente, malgré la remontée du niveau aval, l'écoulement reste dénoyé avec une veine très rapide et un ressaut énergique à l'aval, ce n'est que

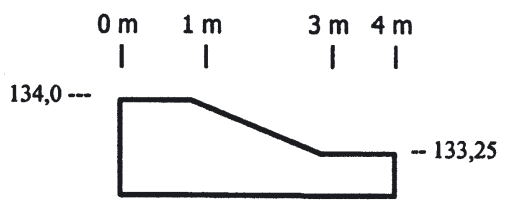

Coupe du seuil coté RD (profil en trapèze) Right bank weir cross section

Tableau II. - Evolution des caractéristiques de l'écoulement au travers du seuil RG

Table II. - Flow characteristics over the right bank weir

\begin{tabular}{|l|ccccc|}
\hline $\begin{array}{l}\text { Distance } \\
\text { comptée depuis } \\
\text { l'amont du seuil }\end{array}$ & $0 \mathrm{~m}$ & $1 \mathrm{~m}$ & $5 \mathrm{~m}$ & $6 \mathrm{~m}$ \\
\hline Débit Loire & \multicolumn{5}{|c|}{ Vitesse $(\mathrm{m} / \mathrm{s})$} \\
\hline $200 \mathrm{~m}^{3} / \mathrm{s}$ & 1,2 à & 2,1 & à & 2,33 à & 4,5 \\
\hline $300 \mathrm{~m}^{3} / \mathrm{s}$ & 1,4 à & 2,3 & à & 2,60 à & 4,3 \\
\hline $600 \mathrm{~m}^{3} / \mathrm{s}$ & 1,8 à & 2,8 & à & 3,10 à & 4 \\
\hline $900 \mathrm{~m}^{3} / \mathrm{s}$ & 2,0 à & 3,1 & à & 3,5 à & 3,5 \\
\hline
\end{tabular}

\begin{tabular}{|c|c|c|}
\hline $\begin{array}{c}\text { Aspect } \\
\text { écoulement } \\
\text { à l'aval }\end{array}$ & $\begin{array}{c}\text { Tirant d'eau } \\
\text { minimum }\end{array}$ & $\begin{array}{c}\text { Chute } \\
\text { tot. }\end{array}$ \\
\hline plongeant & 0,22 & $1 \mathrm{~m}$ \\
\hline plongeant & 0,29 & $0,9 \mathrm{~m}$ \\
\hline horizontal & 0,47 & $0,60 \mathrm{~m}$ \\
\hline horizontal & 0,8 & $0,3 \mathrm{~m}$ \\
\hline
\end{tabular}


Tableau III. - Evolution des caractéristiques de l'écoulement au travers du seuil RD.

Table III. - Flow characteristics over the left bank weir.

\begin{tabular}{|l|c|ccc|c|c|}
\hline $\begin{array}{l}\text { Distance } \\
\text { comptée depuis } \\
\text { l'amont du seuil }\end{array}$ & $0 \mathrm{~m}$ & $1 \mathrm{~m}$ & $3 \mathrm{~m}$ & $4 \mathrm{~m}$ \\
\hline Débit Loire & \multicolumn{5}{|c|}{ Vitesse (m/s) } \\
\hline $200 \mathrm{~m}^{3 / \mathrm{s}}$ & 1,0 à & 1,6 & à & 4,6 & 4,6 \\
\hline $300 \mathrm{~m}^{3 / \mathrm{s}}$ & 1,24 à & 1,9 & à & 4,9 & 4,9 \\
\hline $600 \mathrm{~m}^{3 / \mathrm{s}}$ & 1,7 à & 2,5 & à & 5,2 & 5,2 \\
\hline $900 \mathrm{~m}^{3 / \mathrm{s}}$ & 1,9 à & 2,9 & à & 5 & $5 ?$ \\
\hline
\end{tabular}

\begin{tabular}{|c|c|c|}
\hline $\begin{array}{c}\text { Aspect } \\
\text { écoulement } \\
\text { à l'aval }\end{array}$ & $\begin{array}{c}\text { Tirant } \\
\text { d'eau } \\
\text { minimum }\end{array}$ & $\begin{array}{c}\text { Chute } \\
\text { tot. }\end{array}$ \\
\hline $\begin{array}{c}\text { jet horizontal, } \\
\text { ressaut à l'aval }\end{array}$ & 0,09 & $1 \mathrm{~m}$ \\
\hline $\begin{array}{c}\text { jet horizontal, } \\
\text { ressaut à l'aval }\end{array}$ & 0,14 & $0,9 \mathrm{~m}$ \\
\hline $\begin{array}{c}\text { jet horizontal, } \\
\text { ressaut à l'aval }\end{array}$ & 0,29 & $0,60 \mathrm{~m}$ \\
\hline $\begin{array}{c}\text { ressaut sur le } \\
\text { plan incliné ? }\end{array}$ & 0,5 & $0,3 \mathrm{~m}$ \\
\hline
\end{tabular}

Iorsque la chute devient inférieure à $0,3 \mathrm{~m}$ que le seuil s'ennoie et que les vitesses diminuent nettement.

Par ailleurs, l'écoulement sur ces seuils en béton présente une grande homogénéité ; lorsque les conditions sont impropres au passage, il existe peu de discontinuité où les poissons peuvent profiter de conditions locales plus favorables.

\subsection{Importance de l'obstacle pour la migration}

Malgré ces éléments défavorables, la diminution de la chute avec l'augmentation de débit permet à tous les seuils de devenir franchissables pour un débit suffisamment haut (plus élevé à Saint-Laurent qu'à Dampierre ou Belleville). Mais plus ce débit est élevé, et plus il est rare, l'impact sur la population doit donc s'apprécier à travers la fréquence des débits permet- tant le passage pendant la période de migration.

Outre les conditions hydrauliques sur l'obstacle, les possibilités de franchissement dépendent de nombreux autres facteurs biologiques ou environnementaux: espèce, stade de développement, état physiologique, température de l'eau... A un débit donné, l'obstacle peut ainsi être partiel dans la mesure où seule une fraction de la population parvient à passer, ou bien le passage s'effectue, mais après un temps d'attente ou de recherche plus ou moins long.

Dans ces conditions, il est difficile de définir un débit précis à partir duquel un obstacle est effacé. Cette restriction étant apportée, il est néanmoins instructif de comparer les vitesses d'écoulement sur le seuil aux capacités de nage des poissons pour avoir une idée de l'acuité du problème posé par l'obstacle. 
Tableau IV. - Capacité de nage de quelques espèces, d'après M. Larinier et al. (1995).

Table IV. - Swimming velocity for some fish species, from M. Larinier and al. (1995)

\begin{tabular}{|c|c|c|}
\hline $\begin{array}{c}\text { Grands salmonidés } \\
\text { migrateurs } \\
\text { (saumons, truites de mer) }\end{array}$ & $\begin{array}{l}\text { Truites et cyprinidés } \\
\text { rhéophiles }\end{array}$ & Aloses \\
\hline Vitesse de pointe : 6 à $8 \mathrm{~m} / \mathrm{s}$ & Vitesse de pointe : 3 à $4 \mathrm{~m} / \mathrm{s}$ & Vitesse de pointe : 4 à $5 \mathrm{~m} / \mathrm{s}$ \\
\hline Vitesse sur $3 \mathrm{~m}$ : & Vitesse sur $3 \mathrm{~m}: \quad 2,5 \mathrm{~m} / \mathrm{s}$ & Vitesse sur $3 \mathrm{~m}$ : \\
\hline Vitesse sur $5 \mathrm{~m}$ : & Vitesse sur $5 \mathrm{~m}$ : & Vitesse sur $5 \mathrm{~m}$ : \\
\hline Vitesse sur $10 \mathrm{~m}$ : & Vitesse sur $10 \mathrm{~m}: \quad 1,2 \mathrm{~m} / \mathrm{s}$ & Vitesse sur $10 \mathrm{~m}$ : \\
\hline Tirant d'eau minimum : $0,20 \mathrm{~m}$ & Tirant d'eau minimum : 0,10 m & Tirant d'eau minimum : 0,25 m \\
\hline
\end{tabular}

Les capacités de nage des poissons ne sont bien documentées que pour les salmonidés et les aloses; le Tableau IV regroupe des données rassemblées par $\mathrm{M}$. Larinier et al. (1995). Il donne les vitesses maximales d'écoulement compatibles avec le passage des poissons. Ces vitesses décroissent avec la distance à parcourir.

Ainsi, si l'on compare ces données aux caractéristiques de l'écoulement évaluées sur le seuil de Belleville, on peut avancer les conclusions suivantes pour le passage des aloses:

- Le seuil trapézoïdal (rive droite) est pratiquement infranchissable jusqu'à des débits élevés en raison des fortes vitesses et des tirants d'eau réduits.

- Pour le seuil plat, en rive gauche, les vitesses et tirant d'eau sur le seuil lui-même deviennent compatibles avec les capacités de nage des aloses entre 200 et $600 \mathrm{~m}^{3} / \mathrm{s}$ (vitesse de 4 à $4,5 \mathrm{~m} / \mathrm{s}$ en pointe puis vitesse de 1,2 à $3,1 \mathrm{~m} / \mathrm{s}$ sur $5 \mathrm{~m}$ ). Le passage est alors plutôt conditionné par l'allure de l'écoule- ment à l'aval immédiat du seuil : écoulement plongeant peu favorable; ou horizontal plus favorable, la transition se situant vers $400 \mathrm{~m}^{3} / \mathrm{s}$.

Enfin, une autre source d'information sur l'importance de l'obstacle résulte des suivis régionaux de la migration: comptage des pêches, observation de passages ou d'accumulations en d'autres points du cours d'eau, observations des frayères... Une synthèse de tous ces éléments d'appréciation et des données des suivis disponibles a été tentée pour le seuil de la centrale de Belleville, qui est équipé actuellement de passes à bassins à fentes verticales par JP Bouchard et al. (2000). L'étude conclut que le franchissement du seuil par les saumons, essentiellement en dehors des passes, a été constaté dans une gamme de débit de 200 à $700 \mathrm{~m}^{3} / \mathrm{s}$, le retard occasionné allant de quelques jours jusqu'à 50 jours pour certains individus. Pour l'alose, les suivis montrent que des franchissements ont lieu tous les ans, avec des accumulations d'alose au bar- 
rage de Decize, en amont de Belleville, mais en quantités très variables. En 1999, ils se seraient produits pour des débits compris entre 250 et $35 \mathrm{~m}^{3} / \mathrm{s}$. Mais dans l'ensemble, on ne peut évaluer quelle fraction de la population parvient effectivement à passer.

\section{LA PROBLÈMATIQUE DU FRANCHISSEMENT}

Pour permettre le passage des poissons, il faut fractionner la chute et donc ménager un ou des bassins intermédiaires où l'agitation soit suffisamment faible pour que les poissons puissent se maintenir avant de passer la chute suivante. L'agitation dans les passes est directement liée au débit utilisé ; plus il est important, plus la passe doit être volumineuse pour conserver une agitation acceptable. Par contre, sur des seuils larges comme ceux de la Loire (de 200 à $400 \mathrm{~m}$ de large), le débit réduit d'une passe à poissons sera difficilement perçu par les migrateurs se présentant loin de l'entrée. La conception d'ouvrages de franchissement sur la Loire se heurte donc à cette contradiction: ménager des conditions confortables de passage dans des ouvrages peu spécifiques, donc avec des débits faibles à l'échelle de la rivière, mais alors risquer que l'entrée de la passe soit difficilement trouvée. Face à cette alternative, les choix ont évolué au cours du temps.

\section{1 Évolution historique des solutions}

Même si tous les seuils comportaient lors de leur construction un dispositif rustique de passage, il s'agissait d'une simple échancrure destinée à concentrer l'écoulement en étiage, mais tout à fait insuffisante compte tenu de la hauteur de chute actuelle. Les premiers dispositifs modernes sont les grandes passes à bassins successifs conçues en 1980 pour le site de Belleville.

Parmi toutes les espèces susceptibles de migrer sur la Loire, l'alose a été retenue comme espèce de référence pour la conception des ouvrages de franchissement. En effet, ses exigences et sa capacité de nage réduite par rapport aux salmonidés permettent de garantir l'efficacité de la passe pour tous les grands migrateurs lorsque son efficacité est assurée pour l'alose. Les passes à bassins dimensionnées pour les aloses s'avèrent également permettre le passage de nombreux cyprinidés rhéophiles, seule l'anguille, a imposé plus récemment, des contraintes spécifiques.

\subsubsection{Passes à bassins de Belleville}

Les deux grandes passes à bassins de plus de $80 \mathrm{~m}$ de long ont été construites au droit du seuil de Belleville, en rive droite et à $100 \mathrm{~m}$ de la rive gauche. Elles sont visibles sur les Photos 2 et 3.

Lors de la conception de ces ouvrages en 1980, les connaissances 

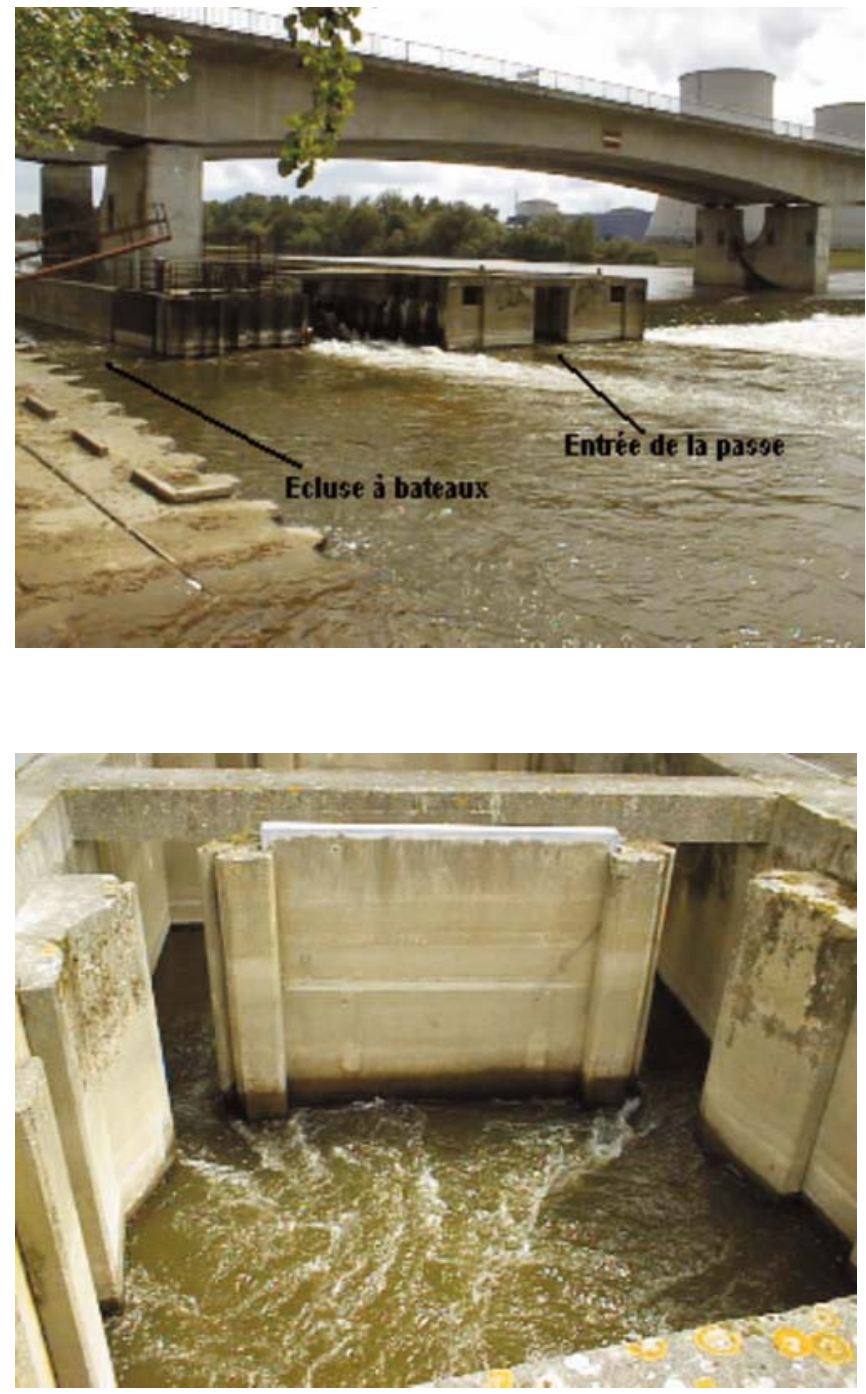

Photo 2. - Passe à bassins de Belleville (RD) vue de l'aval.

Photo 2. - Belleville fish pass

Photo 3. - Passe à bassins de Belleville, vue d'un bassin.

Phtoto 3. - Belleville fish pass, pool with vertical slots.

réduites des capacités de nage et des besoins spécifiques de l'alose avaient amené à prévoir des chutes faibles de $18 \mathrm{~cm}$ entre les bassins, pour une chute totale anticipée sur le seuil de $3 \mathrm{~m}$. Les ouvrages résultants sont donc de très grande longueur.
Malgré ses dimensions imposantes et l'ajout de débit d'attrait dans le bassin aval, le débit écoulé dans chaque passe restait modéré vis-àvis du débit total de la Loire et de la largeur du seuil (Tableau V). 
Tableau V. - Débits dans les passes à bassins de Belleville.

Table V. - Water discharge in the Belleville fish pass.

\begin{tabular}{|c|c|c|c|c|}
\hline $\begin{array}{c}\text { Débit Loire } \\
\left(\mathrm{m}^{3} / \mathrm{s}\right)\end{array}$ & Débit bassins & Débit d'attrait & $\begin{array}{c}\text { Débit total } \\
(2 \text { passes })\end{array}$ & $\%$ débit Loire \\
\hline 100 & 2 & 0,3 & 4,6 & $4,6 \%$ \\
\hline 300 & 2,8 & 1,8 & 9,2 & $3 \%$ \\
\hline 600 & 3,6 & 4 & 15,2 & $2,5 \%$ \\
\hline
\end{tabular}

Les premiers suivis des passes ont révélé la grande diversité des espèces présentes (16 espèces inventoriées) dont certaines à faible capacité de nage (ablette, tanche...), prouvant que les conditions hydrauliques y sont effectivement très confortables. La dissipation volumique d'énergie dans chaque bassin, permet d'apprécier l'agitation qui y règne. Dans ces passes, ce paramètre s'établit à $128 \mathrm{~W} / \mathrm{m}^{3}$.

Par contre, le nombre très réduit d'aloses et de grands migrateurs démontre la difficulté pour ces poissons de trouver l'entrée de la passe. L'attractivité de ces dispositifs est, de plus, réduite par leur localisation éloignée de la berge rive gauche, où se présentent probablement les migrateurs. Cette solution d'aménagement s'avère donc privilégier exagérément le confort des conditions hydrauliques dans la passe au détriment de son attractivité.

\subsubsection{Grands bassins en bétons}

Pour le seuil de Saint-Laurent, on rechercha donc un concept assurant une meilleure attractivité avec une passe écoulant un débit important dans un nombre réduit de bassins. La passe se composait de deux grands bassins implantés le long de la rive gauche, de dimension $20 \mathrm{~m}$ sur $16 \mathrm{~m}$ et séparés par des seuils biais (Photo 4). La chute attendue entre chaque bassin était de l'ordre de 0,55 m. Cependant, l'abaissement des fonds de la Loire à l'aval de l'ouvrage a augmenté les chutes entre bassins et particulièrement à l'entrée de la passe.

Le suivi hydraulique de la passe montre les conditions de fonctionnements suivantes (Tableau VI).

Les observations de l'époque permirent de confirmer la bonne attractivité de la passe grâce à son fort débit et la bonne localisation de l'entrée de la passe à $20 \mathrm{~m}$ en aval de l'obstacle. Néanmoins, la forte chute aval sur un seuil incliné en béton lisse ne procurait pas des conditions d'écoulement favorables au passage des aloses jusqu'à des débits élevés de la Loire. Cette passe a donc été modifiée ultérieurement suivant le nouveau concept de passe en enrochements. 


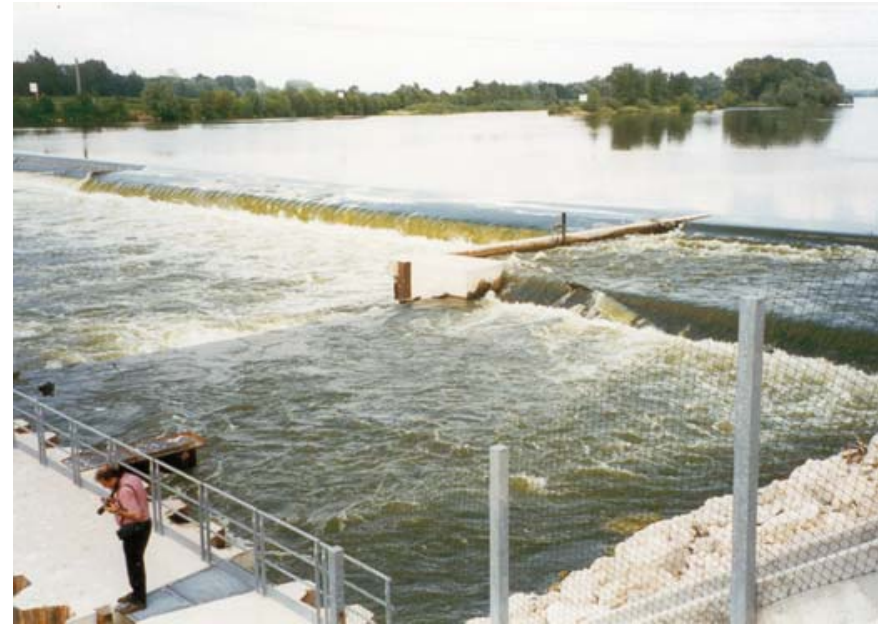

Photo 4 - Saint-Laurent $1^{\text {ere }}$ passe, grands bassins en bétons.

Photo 4 -First implemented fish pass at St Laurent. Fish pass with 2 large concrete pools.

Tableau VI. - Conditions hydrauliques dans les deux grands bassins de la $1^{\text {ère }}$ passe de SaintLaurent.

Table VI. - Flow conditions in the first implemented fish pass at St Laurent.

\begin{tabular}{|c|c|c|c|c|c|c|}
\hline Débit Loire & $\begin{array}{c}\text { Chute 1 } \\
\text { (amont) }\end{array}$ & Chute 2 & $\begin{array}{c}\text { Chute 3 } \\
\text { (aval) }\end{array}$ & $\begin{array}{c}\text { Débit passe } \\
\text { estimé }\end{array}$ & $\begin{array}{c}\% \text { Débit } \\
\text { Loire }\end{array}$ & $\begin{array}{c}\text { Dissipation } \\
\text { d'énergie } \\
\text { bassin 2 }\end{array}$ \\
\hline 100 & 0,43 & 0,60 & 0,75 & 7,8 & $7,8 \%$ & $49 \mathrm{~W} / \mathrm{m}^{3}$ \\
\hline 200 & 0,39 & 0,54 & 0,78 & 17,3 & $5.2 \%$ & $90 \mathrm{~W} / \mathrm{m}^{3}$ \\
\hline 300 & 0,38 & 0,48 & 0,75 & 26,4 & $4,4 \%$ & $115 \mathrm{~W} / \mathrm{m}^{3}$ \\
\hline
\end{tabular}

\subsubsection{Le concept actuel : la passe en enrochements}

\subsubsection{Caractéristiques}

Le concept développé actuellement est issu des travaux expérimentaux de M. Larinier sur les seuils en rivière directement franchissables par les poissons. II conserve le principe du type précédent de passes à poissons et utilise un débit important, dans un nombre réduit de bassins pour conserver une bonne attractivi- té. Par contre, les conditions de passage sont améliorées par l'utilisation de seuils en enrochements.

Ce type de passe a tout d'abord été implanté au seuil de Dampierre en 1998, où deux passes en enrochements ont été construites sur chaque rive, puis pour la modification de la passe de Saint-Laurent en 2000.

Ces passes se composent de grands bassins d'environ $20 \mathrm{~m}$ sur $20 \mathrm{~m}$ séparés par des seuils en enro- 

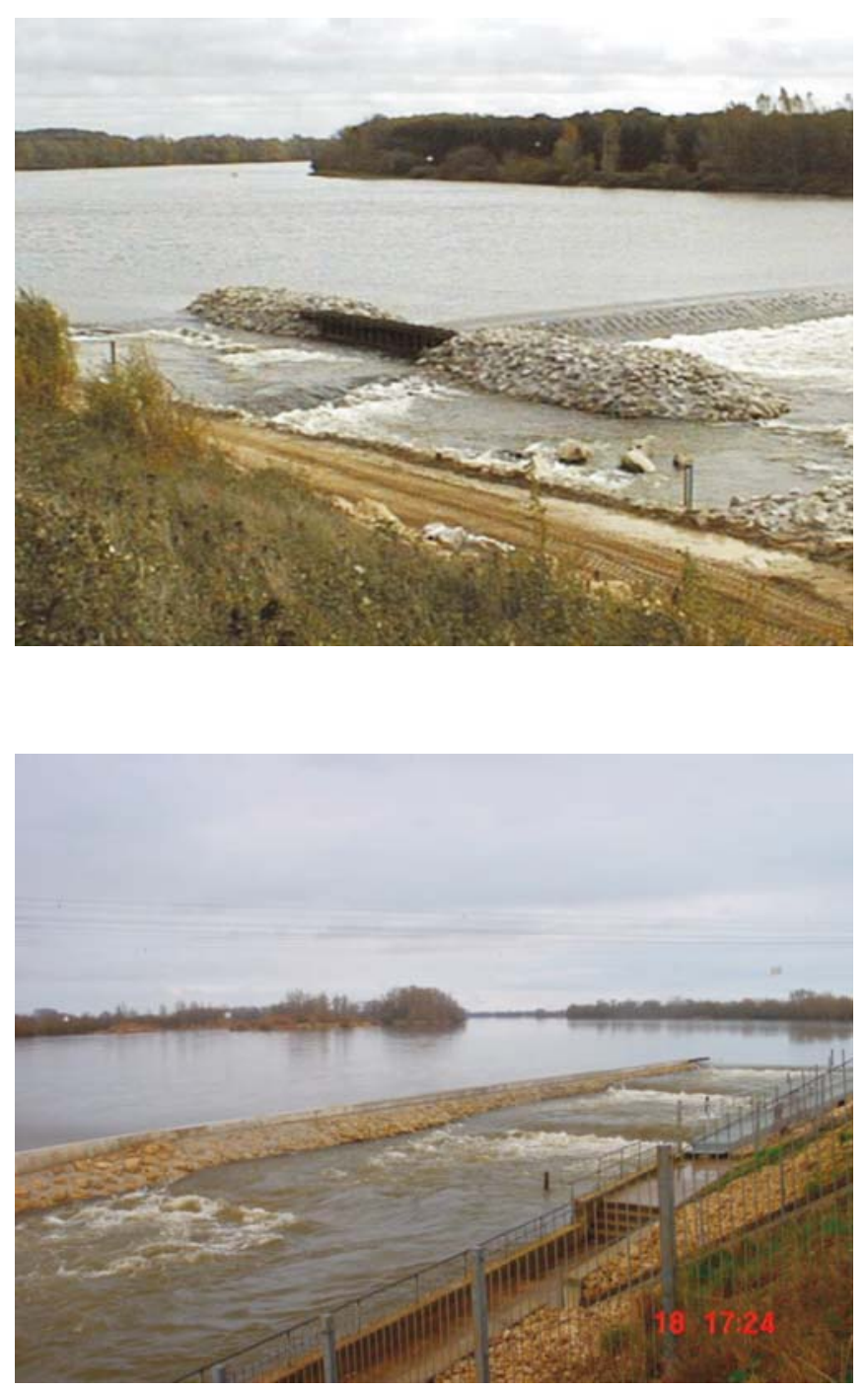

Photo 5. - Passe en enrochements, Dampierre RG.

Photo 5. - Rockfil fish pass at Dampierre on the river Loire.

Photo 6. - Passe en enrochements, Saint-Laurent.

Photo 6. - New rockfil fish pass, St Laurent. chements, les seuils sont biais de façon à présenter une alimentation variable transversalement et donc des tirants d'eau et des vitesses diversifiées. Cette hétérogénéité des conditions est renforcée par l'irrégularité procurée par les enrochements.
Les Photo 5 et 6 illustrent ce type de passe.

Les seuils sont précédés à l'aval par une rampe enrochée avec une pente de $15 \%$, dont la rugosité participe à ralentir l'écoulement. Les autres talus ont une pente de 2/1. 
Les enrochements sont jointoyés de béton jusqu'au milieu de la dernière couche de blocs de façon à garder une forte rugosité mais en limitant la percolation entre les blocs. Les chutes entre bassins varient entre $0,4 \mathrm{~m}$ et $0,6 \mathrm{~m}$.

Signalons enfin que la réalisation de ces ouvrages est très délicate, une précision sur les cotes de $5 \mathrm{~cm}$ est demandée pour la réalisation de la crête des seuils entre bassins, alors qu'ils doivent être construits avec des blocs de plus de $50 \mathrm{~cm}$ de diamètre.

\subsubsection{Fonctionnement hydraulique}

Les seuils biais permettent d'obtenir un débit unitaire (débit par unité de longueur) variable au long du seuil. Pour les aloses, on recherche un débit unitaire compris entre 0,6 et $1 \mathrm{~m}^{3} / \mathrm{s}$ $/ \mathrm{m}$. Selon le niveau amont, la gamme de débit unitaire convenable se déplace transversalement sur le seuil. Les conditions hydrauliques calculées dans la passe de SaintLaurent permettent de vérifier que la gamme optimale est toujours trouvée quel que soit le débit en Loire (Tableau VII).
Les observations du comportement des poissons dans la passe de Dampierre, ont montré que le frottement le long des talus latéraux de la passe, jouait également un grand rôle pour la progression des poissons, en prodiguant une zone de cheminement aisée jusqu'aux abords de la chute. Ce fait a été mis à profit pour la réalisation suivante à Saint-Laurent. On a essayé lors de l'étude sur modèle, d'amplifier le décollement de l'écoulement le long de la paroi, en disposant une tranchée de $0,5 \mathrm{~m}$ de profondeur orientée obliquement par rapport à l'axe de la passe. L'effet est bien visible sur la Photo 7 suivante.

\subsubsection{Efficacité}

Le piégeage étant impossible dans ce type d'ouvrage, on ne dispose pas de véritable suivi quantifié des franchissements. On doit pour le moment se contenter d'observations ponctuelles. Les débits transités et la localisation de l'entrée des passes en limite de la zone perturbée, à $20 \mathrm{~m}$ en aval de l'obstacle (Photo 8), garantissent une bonne attractivité de la passe. Des passages d'aloses ont été observés dans la passe de Dampierre en rive gauche et rive droite.

Tableau VII. - Conditions hydrauliques dans la passe de Saint-Laurent après rénovation.

Table VII. - Flow conditions in the new fish pass at St Laurent.

\begin{tabular}{|c|c|c|c|c|c|c|}
\hline $\begin{array}{c}\text { Débit } \\
\text { Loire } \\
\left(\mathrm{m}^{3} / \mathrm{s}\right)\end{array}$ & $\begin{array}{c}\text { Débit dans } \\
\text { la passe } \\
\left(\mathrm{m}^{3} / \mathrm{s}\right)\end{array}$ & $\begin{array}{c}\% \\
\text { du débit } \\
\text { Loire }\end{array}$ & $\begin{array}{c}\text { Débit } \\
\text { unitaire max. } \\
\left(\mathrm{m}^{3} / \mathrm{s} / \mathrm{m}\right)\end{array}$ & $\begin{array}{c}\text { Débit } \\
\text { unitaire mini } \\
\left(\mathrm{m}^{3} / \mathrm{s} / \mathrm{m}\right)\end{array}$ & $\begin{array}{c}\text { Chute } \\
\text { max. } \\
(\mathrm{m})\end{array}$ & $\begin{array}{c}\text { Dissipation } \\
\text { volumique } \\
\left(\mathrm{W} / \mathrm{m}^{3}\right)\end{array}$ \\
\hline 100 & 3 & $3 \%$ & 0,63 & 0 & 0,45 & 50 \\
\hline 300 & 12 & $4 \%$ & 1,56 & 0,23 & 0,45 & 130 \\
\hline 600 & 25 & $4,2 \%$ & 2,7 & 0,95 & 0,50 & 200 \\
\hline
\end{tabular}



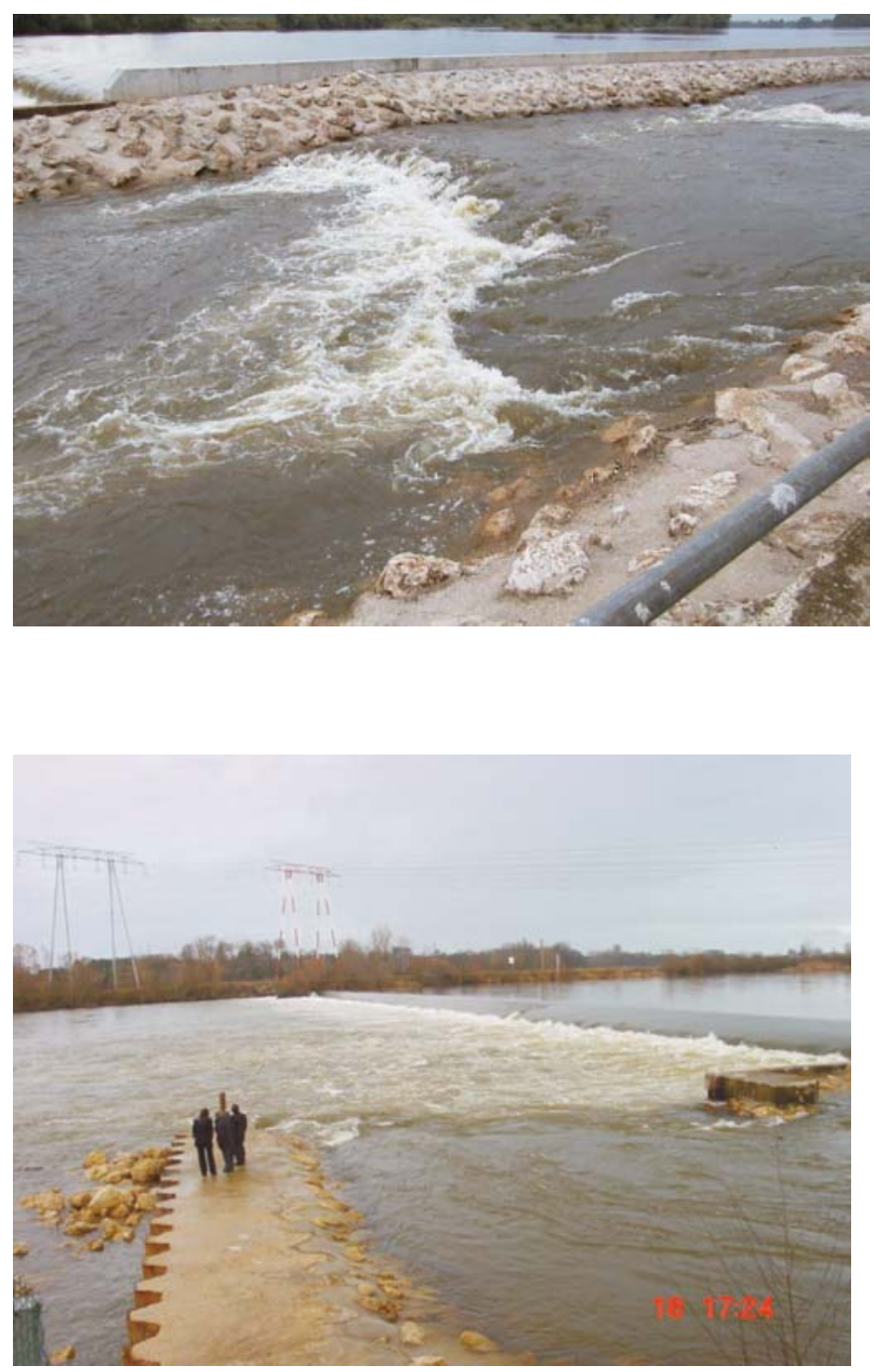

Photo 7. - Saint-Laurent : Ecoulement oblique sur un seuil ménageant une zone de passage privilégiée le long de la paroi.

Photo 7. - Saint-Laurent: transvers side stream on a weir for a better fish progression.

Photo 8. - Saint-Laurent: Entrée de la passe à l'aval.

Photo 8. - Saint-Laurent: Fish pass entrance.
On a trouvé dans les bassins des cyprinidés d'eau calme (Bouvières), ce qui démontre que malgré les chutes relativement importantes, l'hétérogénéité des vitesses près des enrochements permet à de nombreu- ses espèces de traverser l'ouvrage. Enfin, notons que la rugosité des fonds devrait également procurer un substrat favorable à la reptation des anguilles et à la progression des lamproies. 


\section{NOUVEL AMENAGEMENT DU SEUIL DE BELLEVILLE}

On a vu précédemment que les passes à bassins existantes à Belleville offraient de bonnes conditions de passage notamment pour les cyprinidés, mais s'avéraient insuffisamment attractives pour les grands migrateurs.

A Belleville, le lit est exceptionnellement large, mais les migrateurs se présentent préférentiellement dans le chenal rive gauche. Le nouvel aménagement vise donc à fournir un passage sur ce côté de la Loire.
L'installation d'une passe à bassins en enrochements du même type qu'à Dampierre est impossible en raison de la présence du dispositif de rejet des eaux de refroidissement de la centrale dans le seuil lui-même qui ne laisse qu'un espace réduit de $6 \mathrm{~m}$ le long de la rive, insuffisant pour installer une passe à bassins en enrochements.

La solution proposée a donc consister à réaliser un grand pré barrage enroché d'une centaine de mètres de large, situé à $30 \mathrm{~m}$ en aval du seuil de la centrale et barrant tout le chenal. La Photo 9 montre la position du nouveau dispositif proposé.

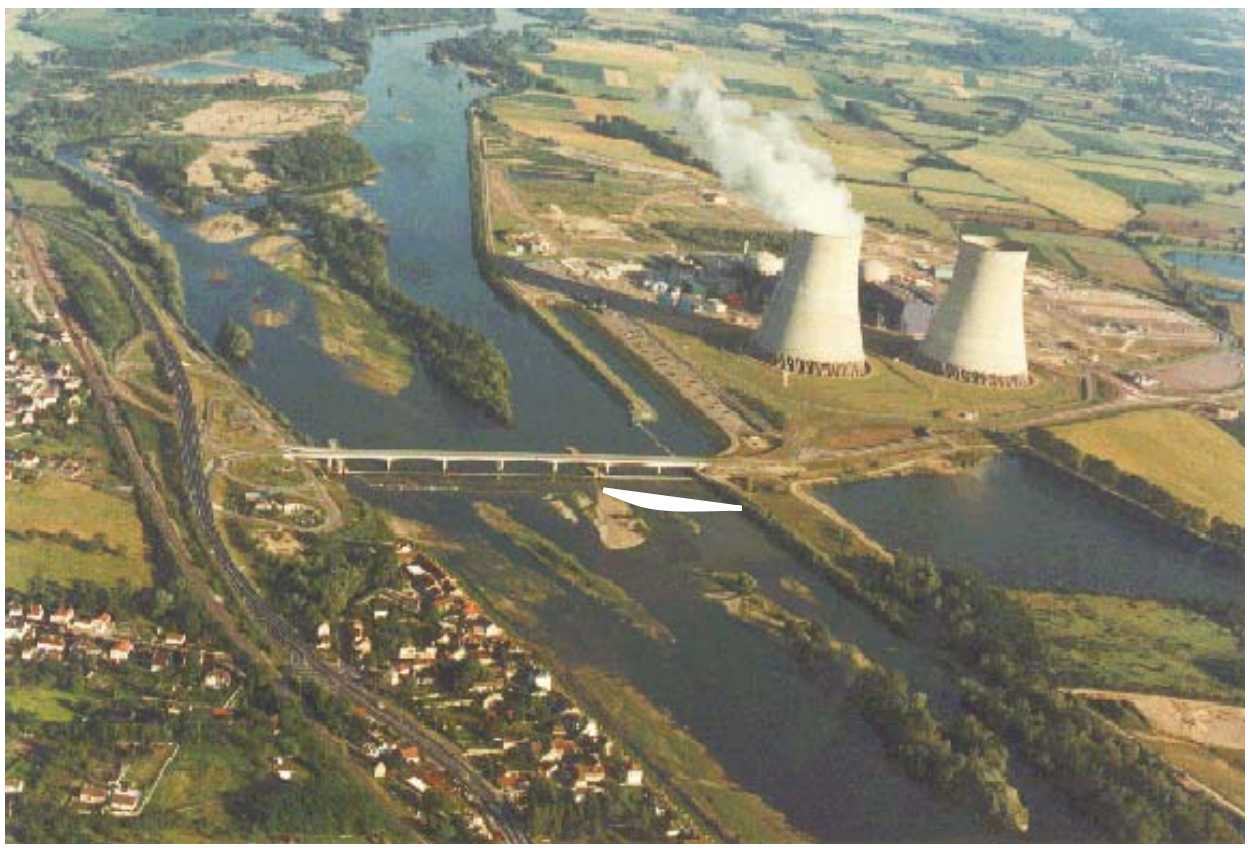

Photo 9 - Belleville : Le trait blanc figure le pré barrage prévu.

Photo 9 - Belleville plant aerial view. The white line shows the planned rockfil weir. 


\subsection{Principes}

La hauteur de chute maximale considérée ici est de $1,35 \mathrm{~m}$. Les caractéristiques du seuil en béton de la centrale, et notamment son épaisseur, sont telles que son franchissement ne serait possible qu'avec une chute réduite à $0,3 \mathrm{~m}$. La chute préalable à franchir sur le pré barrage serait donc de l'ordre de $1 \mathrm{~m}$.

Le pré barrage présente l'avantage de résoudre parfaitement le problème de l'attractivité de la passe, puisque dans ce cas, le franchissement s'effectue sur toute la largeur du chenal. En outre, sa forme permet de guider les poissons vers la passe à bassins et ainsi de donner une nouvelle attractivité à cet ouvrage ancien, dont le confort pour les petites espèces a été démontré. Par contre, la hauteur de chute à franchir sur le pré barrage lui-même est importante.

La solution en cours d'étude s'inspire des principes mis en œuvre pour les passes à bassins en enrochements. La crête du pré barrage n'est pas uniforme mais comporte une partie surbaissée bordée de parties plus élevées pour présenter des zones de conditions hydrauliques contrastées. La rampe aval est faiblement pentue en raison de la hauteur de chute importante à franchir et de la nécessité d'atteindre des vitesses d'écoulement réduites, lorsque la distance à franchir est longue.

\subsection{Moyens d'étude}

Le caractère novateur de la solution envisagée a justifié sa mise au point à l'aide d'un modèle physique hydraulique. Le modèle est à l'échelle du $1 / 15^{\text {éme }}$, il reproduit le chenal rive gauche de la Loire sur $150 \mathrm{~m}$ de large et s'étend à $50 \mathrm{~m}$ à l'amont du seuil et $100 \mathrm{~m}$ à l'aval. Ce modèle a permis de définir les caractéristiques précises du seuil: pente de la rampe aval, forme et profil transversal de la crête, tels qu'il existe pour tous les débits du fleuve une zone du seuil où les vitesses d'écoulement soient compatibles avec les capacités de nages des poissons du Tableau IV.

\section{CONCLUSIONS}

La position des seuils de centrale en Loire moyenne et le contrôle qu'ils exercent sur l'accès aux zones de reproduction, rendent le franchissement de ces obstacles particulièrement essentiel. La mise au point d'ouvrages de franchissement se heurte à la contradiction entre la recherche d'une bonne attractivité de la passe, qui implique de mobiliser un débit important, et la nécessité de conserver dans la passe des conditions hydrauliques assez douces pour que les poissons puissent progresser vers l'amont. Le point d'équilibre entre ces deux contraintes est difficile à trouver dans la mesure où le comportement des poissons dans un nouveau type d'ouvrage est lui-même difficile à mettre en évidence sans uti- 
liser les techniques compliquées du radio pistage.

Cela explique qu'une solution définitive n'a pu être trouvée au premier essai. Les concepts ont évolué faisant du même coup progresser la connaissance du comportement des poissons sur les obstacles à la migration.

L'équipement du seuil de Belleville prévu en 2003, après la rénovation de la passe de Saint-Laurent, et l'équipement du seuil de Dampierre, achèvera la levée des obstacles associés à ces centrales. II marquera une étape importante dans le processus de libération de la circulation des migrateurs sur le bassin de la Loire.

\section{RÉFÉRENCES}

BIBLIOGRAPHIQUES

Bouchard J-P. \& Travade F., 2000 - Examen des possibilités de franchissement du seuil de Belleville par les poissons migrateurs. Rapport EDF HP 73/2000/004, (2000)

Larinier M., Chorda J. \& Ferlin O., 1995 Le franchissement des seuils en enrochements par les poissons migrateurs. Etude expérimentale. Rapport GHAAPPE.

Steinbach P., 2002 - Effets cumulés sur les poissons migrateurs, état et restauration des grands axes de migration du bassin de la Loire. Revue d'hydroécologie appliquée : Tome 13 -Volume 2. 
